\title{
Médula espinal amarrada. Reporte de caso
}

\section{Case Report: Tethered cord syndrome}

Ignacio Cuevas ${ }^{1}$, Patricia Perillo²

\begin{abstract}
We present the case of a 31 year-old patient recent diagnosed with tethered cord syndrome, by MRI, before urgent caesarian section. The image shows the conus medullaris in a low location, reaching L5-S1, which could lead to potential neurological damage if it hadn't been diagnosed. Tethered cord syndrome is a condition in which the spinal cord is enlongated and in a low location, attached to an inelastic structure that holds the conus medullaris, blocking its normal ascension during growth[1]. As a result, the conus medullaris is located below L2 vertebral body, increasing the risk of suffering direct neural damage at spinal anesthesia[1]. Even if direct cord injury can be avoided, the injection of a local anesthetic agent may increase the subarachnoidal preassure leading undirectly to spinal cord damage[2],[4]. The tethered cord is considered to be a contraindication for spinal anesthesia[1],[2],[4]. The incidence of tethered cord is unknown, it can be asymptomatic in adults or present non-specific symptoms, making it difficult to detect before anesthesia[1],[2]. The MRI is the best method for adult diagnosis, allowing us to evaluate the conus medullaris location, injuries and deformations ${ }^{1}$. For newborn diagnosis, ultrasound is an effective method[1],[3].
\end{abstract}

\section{RESUMEN}

Presentamos el caso de una paciente de 31 años coordinada para cesárea de urgencia con diagnóstico reciente de médula amarrada (MA) por resonancia magnética. La imagen muestra el cono medular descendido hasta por lo menos L5-S1, exponiéndola a un potencial daño neurológico de no contar con el diagnóstico. Médula amarrada es una condición en la cual la médula espinal se encuentra estirada y descendida por una estructura inelástica que amarra

\section{Key words:}

Tethered cord syndrome, dysraphism, spinal anesthesia

\section{Palabras clave:}

Síndrome médula amarrada,

disrafismos medulares, anestesia raquídea

1 Residente de Anestesiología, Departamento y Cátedra de Anestesia, Hospital de Clínicas, Universidad de la República, Montevideo, Uruguay.

2 Anestesióloga-Analgesia obstétrica en Hospital Británico Montevideo, Uruguay.

Fecha de recepción: 28 de agosto de 2018

Fecha de aceptación: 12 de diciembre de 2018

ORCID

https://orcid.org/0000-0001-8673-1506

Correspondencia:

Dr. Ignacio Cuevas

Email: iscuevas@gmail.com 
el cono medular, evitando su normal ascenso durante el crecimiento[1]. Como resultado, el cono medular se encuentra por debajo del cuerpo de $\mathrm{L} 2$, incrementando el riesgo de lesión directa con la aguja de raquianestesia[1]. Incluso en aquellos pacientes en que la lesión directa no se produce, la inyección del anestésico local puede aumentar la presión subaracnoidea pudiendo provocar una lesión indirecta[2],[4]. La MA es considerada una contraindicación para la anestesia raquídea[1],[2],[4]. La incidencia de MA se desconoce, en adultos puede ser asintomática o presentar síntomas inespecíficos dificultando su diagnóstico preoperatorio[1],[2]. La RNM es el método de elección para el diagnóstico en adultos, permitiéndonos valorar la localización del cono medular, lesiones y malformaciones[1]. En neonatos la ultrasonografía puede ser también de utilidad para el diagnóstico[1],[3].

\section{Introducción}

P resentamos el caso de una paciente de 31 años coordinada para cesárea de urgencia por trabajo de parto detenido. En el preoperatorio inmediato nos enfrentamos al diagnóstico reciente de médula amarrada por lipoma sacro. Paciente asintomática, con el antecedente personal de haber sido operada al año de vida por la misma patología.

Médula amarrada es una condición en la cual la médula espinal se encuentra estirada y descendida por una estructura inelástica que amarra el cono medular, evitando su normal ascenso durante el crecimiento ${ }^{1}$. Como resultado, el cono medular se encuentra por debajo del cuerpo de L2, incrementando el riesgo de lesión directa con la aguja de raquianestesia[1].

\section{Reporte de caso}

Paciente de 31 años coordinada para cesárea de urgencia por trabajo de parto detenido.

Como antecedente anestésico-quirúrgico refiere cirugía al año de vida por lipoma sacro.

AGO: Primigesta. Cursando 39 semanas de embarazo bien controlado y tolerado. Ingresa para inducción, indicándose finalmente cesárea de urgencia por trabajo de parto detenido.

Al examen físico: buena apertura bucal, mallampati 1, flexo-extensión y distancias normales.

A la inspección de la columna se evidencia cicatriz en línea media a nivel lumbosacro. Sin otra particularidad a destacar.

En la evaluación preoperatoria inmediata, paciente relata cirugía al año de vida por lipoma sacro, y resonancia nuclear magnética (RNM) reciente que informa: médula amarrada con lipoma sacro. Protrusión discal L5-S1.

Clínicamente asintomática al interrogatorio. Niega sintomatología neurológica, dolores lumbares o de mmii y trastornos esfinterianos.

Al observar las imágenes de RNM, evidenciamos el lipoma sacro y la médula amarrada, la cual llega hasta por lo menos L5-S1 y la cola de caballo-filum terminal hasta sacro (Figuras 1 y 2 ).

A pesar de la solicitud de la paciente de querer una técnica regional para poder presenciar el nacimiento de su bebé, luego de explicarle detalladamente el riego-beneficio, y contraindicación de la técnica regional, se decide realizar una anestesia general balanceada. Inducción en secuencia rápida con Propofol

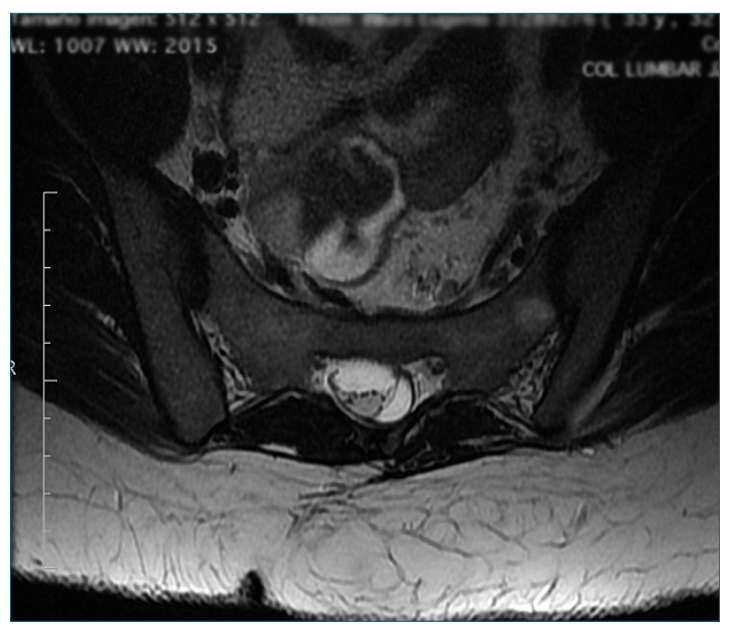

Figura 1. Corte sagital en T2 y T1. 


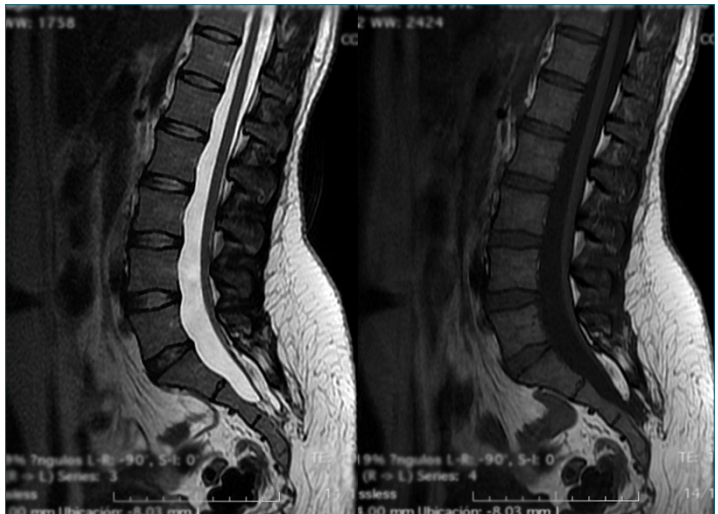

Figura 2. Corte axial. Lipoma sacro amarrando cola de caballo.

$2 \mathrm{mg} / \mathrm{kg}$, succinilcolina $1 \mathrm{mg} / \mathrm{kg}$. Intubación orotraqueal con Sonda número 7 sin incidentes. Recién nacido vivo, apgar 9-10. La paciente se extuba en block y es trasladada a la sala de recuperación pos-anestésica junto a su bebé.

\section{Discusión}

Médula amarrada (MA) es una condición en la cual la médula espinal se encuentra estirada y descendida por una estructura inelástica que amarra el cono medular, evitando su normal ascenso durante el crecimiento. Entre los disrafismos espinales más frecuentemente involucrados se encuentran: mielomeningocele, lipomielomeningocele, diastematomielia, lipomas intradurales y lesiones quísticas[1]. Como resultado, el cono medular se encuentra por debajo del cuerpo de $L 2$, incrementando el riesgo de lesión directa con la aguja de raquianestesia[1].

En nuestra paciente, la imagen de RNM muestra el cono medular llegando hasta por lo menos L5-S1, exponiéndola a un potencial daño neurológico de no contar con el diagnóstico.

La mayoría de los casos de MA por disrafismos medulares son diagnosticados y tratados mediante cirugía en los primeros meses de vida[1]. Nuestra paciente relata una cirugía al año de vida por un lipoma sacro, sin conocer más detalles. Presumimos se le pueda haber desamarrado en esa oportunidad la médula, conducta más frecuente en estos casos.

Por otro lado, los disrafismos espinales ocultos, usualmente pueden asociarse a lesiones en la piel como: angiomas, lipomas subcutáneos o fositas y malformaciones urogenitales[1],[3]. Un paciente pe- diátrico, que presenta una anormalidad urogenital junto a una lesión cutánea menor, debe ser estudiando en busca de un disrafismo oculto[3].

En adultos, los síntomas más comunes que pueden orientarnos a un síndrome de médula amarrada son: déficit neurológico motor y/o sensitivo progresivo y bilateral en miembros inferiores; disminución de reflejos en tendón rotuliano y aquiles; dolores en columna lumbar y miembros inferiores; trastornos urológicos como vejiga neurógena e incontinencia de esfínteres; malformaciones en los pies[1],[2],[4].

La incidencia de MA se desconoce, en adultos puede ser asintomática o presentar síntomas inespecíficos dificultando su diagnóstico preoperatorio[1],[2].

La RNM es el método de elección para el diagnóstico en adultos, permitiéndonos valorar la localización del cono medular, lesiones y malformaciones ${ }^{1}$. En neonatos la ultrasonografía puede ser también de utilidad para el diagnóstico[1],[3].

El tratamiento consiste en desamarrar la médula y extraer la lesión quirúrgicamente. La indicación de cirugía en pacientes asintomáticos como la nuestra, no es tan clara, incluso pudiéndose evitar posibles daños neurológicos a futuro[1].

La raquianestesia en pacientes con MA puede resultar en una lesión directa de la médula espinal. Incluso en aquellos en que la lesión directa no se produce, la inyección del anestésico local puede aumentar la presión subaracnoidea pudiendo causar una lesión indirecta[2],[4]. La MA es considerada una contraindicación para raquianestesia[1],[2],[4].

Las lesiones pueden ser transitorias o permanentes. En la mayoría de los casos son transitorias, debido al edema producido durante la punción. En los casos más graves se puede producir un hematoma que provoque una lesión mecánica por compresión o lesión directa por la aguja provocando daños permanentes.

En aquellos pacientes que presenten síntomas como parestesias y debilidad de miembros inferiores debemos sospechar una lesión por médula amarrada y solicitar una RNM.

Existen pocos casos reportados sobre complicaciones por anestesia raquídea en pacientes con médula amarrada. La mayoría son reportes de casos, en los cuales debido a síntomas neurológicos luego de la raquianestesia, se llega al diagnóstico por RNM.

Un estudio observacional retrospectivo llevado a cabo por la universidad de Xi'an Jiaotong, China, publicado en la revista medicine en 2016, revisó historias clínicas de 28.160 pacientes sometidos a raquianestesia o anestesia combinada epidural + raquídea entre 2008 y 2015, encontrando 4 casos de médula amarrada.

Durante la realización del bloqueo subaracnoi- 
deo, los 4 pacientes presentaron dolor irradiado a los miembros inferiores o región perineal, desapareciendo inmediatamente luego de retirada la aguja. El bloqueo se logró satisfactoriamente y las cirugías fueron llevadas a cabo sin inconvenientes.

Dos - 3 días luego de la anestesia, los pacientes comenzaron con parestesias y debilidad en miembros inferiores, llegándose al diagnóstico de médula amarrada por RNM. El cono medular se encontraba por debajo de L3 en los 4 casos, la presencia de edema medular también presente en todos los pacientes. En el caso 3 el cono medular finalizaba en L5.

Los pacientes fueron tratados con vit-B12, vit-B1 y corticoides. Respecto a la sintomatología neurológica previa, el caso 1 presentaba ocasionalmente dolor severo en el miembro inferior derecho. Casos 2, 3 y 4 sentían ocasionalmente dolor en la espalda y región perineal, agregando además el caso 4 dificultades en la flexión. Todos síntomas como hemos visto anteriormente inespecíficos, que no alertaron a los anestesiólogos actuantes.

Los síntomas neurológicos en los casos 1, 2 y 4 desaparecieron completamente en 3 semanas. En el caso 3, los síntomas persistieron hasta 4 meses luego de la cirugía.

En 2014, el Departamento de Anestesiología del Prebyterian Medical Center, Jeonji, Korea, reporta el caso de una paciente de 52 años, que ingresó al Departamento de Neurología por dolor y alteraciones de la sensibilidad en el miembro inferior derecho. Como antecedente, la paciente había recibido raquianestesia para una cirugía urológica, comenzando con la sintomatología durante la recuperación. Si bien los síntomas mejoraron gradualmente, persistieron durante 2 meses, lo que la llevó a consultar.

Bajo sospecha de lesión medular por anestesia raquídea, se solicitó una RNM diagnosticándose un engrosamiento del filum terminal y siringomielia. El cono medular finalizaba a nivel del cuerpo de L5, por amarramiento del filum terminal engrosado. Se diagnostica probable lesión medular directa por aguja espinal. Luego de 10 meses con tratamiento v/o los síntomas revirtieron completamente.

Otro caso publicado en 2007 en Journal of Anesthesia, por un equipo japonés, reporta el caso de una paciente de 47 años con síndrome de médula amarrada diagnosticado en el preoperatorio de artroscopia de rodilla. Durante el examen neurológico preoperatorio, si bien la paciente no relataba sintomatología, se constató una debilidad en la dorsiflexión y reducción de la movilidad en el cuello de pie y primer dedo. Se identificó además una malformación de "pie en garra" en el miembro inferior izquierdo. En la re- gión sacra se encontró un lipoma con estigma cutáneo.

La RNM mostró una médula amarrada a una masa sacra, la cual estaba comunicada a su vez con una fosita y el lipoma sacro observado en el examen físico.

Una vez realizado el diagnóstico se procedió con la cirugía bajo AGB sin incidentes.

Reporte muy interesante, especialmente por la sospecha al realizar un examen físico neurológico preoperatorio.

El Departamento de Anestesiología de Yonsei University - Seoul, Korea, reporta un paciente de 12 meses coordinado para una pieloureterotomía por hidronefrosis. Luego de realizada una $A G$, se procede a realizar un bloqueo caudal bajo ultrasonografía. Se identifica una fosita interglútea, y mediante la ecografía se constata un filum terminal engrosado, con un cono medular descendido. El bloqueo caudal no es realizado y se procede con la cirugía.

En el postoperatorio se realiza una RNM diagnosticándose un lipoma, indicándose cirugía de desamarramiento un mes después.

En el caso de nuestra paciente, contábamos con el diagnóstico preoperatorio a través de RNM. Al igual que en muchos de los casos reportados, no presentaba sintomatología neurológica, y había consultado con varios colegas de diferentes especialidades en busca de una conducta a tomar frente a una posible anestesia raquídea. Evaluamos el riesgo potencial al que nos enfrentábamos, decidiendo realizar una anestesia general.

La anestesia epidural podría tal vez haber sido una opción, pero nos pareció excesivo el riesgo frente a una punción dural accidental, sabiendo que la médula espinal está amarrada a la pared posterior del conducto raquídeo.

Consultamos al Prof. Agregado. Dr. Carlos Álvarez, referente en analgesia obstétrica de Uruguay, quien respaldo totalmente la conducta tomada, considerando que las técnicas regionales espinales están contraindicadas en una columna patológica como ésta, más allá de los deseos de la paciente. Destacó que en estos casos debe primar la seguridad y el sentido común.

El Dr. Alejandro Corujo, referente en anestesia regional, también considera una contraindicación para técnicas regionales espinales para este tipo de patologías.

No hemos encontrado en la bibliografía opiniones a favor o en contra de la técnica epidural en estos casos, simplemente se contraindica la anestesia raquídea; pero debemos plantearnos ciertas interrogantes: ¿se verá dificultada la técnica por la presencia del li- 
poma? ¿Cuál será el comportamiento de la anestesia epidural en estas condiciones? ¿Se verá alterado el desplazamiento del AL? ¿Qué sucedería frente a un sangrado que provoque un aumento en la presión, en una médula que ya puede tener de cierta forma comprometido su flujo? El mismo anestésico local en una técnica epidural provocará un aumento de la presión.

Seguramente se han colocado catéteres epidurales a pacientes con esta patología sin incidentes, ya que de hecho no encontramos reportes de casos al respecto.

Otro punto importante a tener en cuenta es el riesgo legal que se toma frente a un caso como éste. ¿Qué sucedería frente a una complicación? ¿Estaríamos amparados en caso de una complicación habiendo realizado una técnica neuroaxial, en una columna que de antemano sabemos es patológica?

Pensamos, la opción elegida, es la más prudente y segura tanto para la paciente como para el anestesiólogo actuante.

\section{Conclusiones}

La raquianestesia en pacientes con médula amarrada debe ser evitada dado el riego aumentado de complicaciones neurológicas.

Frente a un paciente con síntomas tales como: dolor de espalda, vejiga neurógena, alteraciones motoras o sensitivas, es importante nos alertemos sobre la posibilidad de un síndrome de médula amarrada.

Un paciente pediátrico, que presenta una anormalidad urogenital junto a una lesión cutánea menor, debe ser estudiado en busca de un disrafismo oculto. Ante síntomas neurológicos luego de una anestesia raquídea debe realizarse una RNM para realizar un diagnóstico etiológico.

La MA es considerada una contraindicación para raquianestesia[1],[2],[4]. No hemos encontrado bibliografía que respalde el uso de una técnica epidural en estos pacientes.

\section{Referencias}

1. Yu Yil Kim, J. W. Song, J. H. Lim, Y. S. Kim, Y. E. Known, J. $H$. Lee. Nerve injury in an undiagnosed adult tethered cord syndrome patients following spinal anesthesia - a case report - Anesthesiology and Pain Med. 2015; 10: 171-174.
2. Liu JJ, Guan Z, Gao Z, Xiang L, Zhao F, Huang SL. Complications after spinal anesthesia in adult tethered cord syndrome. Medicine (Baltimore). 2016 Jul;95(29):e4289.

3. Kim J, Shin S, Lee H, Kil HK. Tethered spinal cord syndrome detected during ultrasound for caudal block in a child with single urological anomaly. Korean J Anesthesiol. 2013 Jun;64(6):5523.

4. Yokoyama T, Sadahiro T, Sluka KA, Yamashita K, Tokoroyama $\mathrm{H}$, Manabe M. Tethered cord syndrome discovered in preoperative examination. J Anesth. 2007;21(2):270-2. 